\title{
Nitro-oleic Acid Decreases Transcription of the Angiotensin II Type I Receptor Gene in Aortic Smooth Muscle Cells
}

\author{
Huan Wang, Hongsheng Ouyang, Yaping Tian, Zhuang Li, Xiaolei Han, Xingxing Liu, Guangyao Ran, \\ Gangqi Wang, Daxin Pang, and Xiaochun Tang
}

Received: 10 April 2014 / Revised: 22 June 2014 / Accepted: 28 June 2014

(C) The Korean Society for Biotechnology and Bioengineering and Springer 2015

In the 2014 issue of Biotechnology and Bioprocess Engineering (BBE), an error occurred in the research article: Huan Wang, Hongsheng Ouyang, Yaping Tian, huang Li, Xiaolei Han, Xingxing Liu, Guangyao Ran, Gangqi Wang, Daxin Pang, and Xiaochun Tang (2014) Nitro-oleic Acid Decreases Transcription of the Angiotensin II Type I Receptor Gene in Aortic Smooth Muscle Cells. Biotechnol. Bioprocess Eng. 19: 740-746.

\section{- In the authors's affiliation}

\section{Original wording:}

Huan Wang

Department of Bioengineering, Collage of Jilin Agricultural Science and Technology, Jilin University, Jilin 130062, China

\section{This should be replaced by:}

Huan Wang

Department of Bioengineering, Jilin Agriculture Science and Technology College, Jilin 132101, China

Received: 10 March 2015

Huan Wang ${ }^{\dagger}$, Hongsheng Ouyang ${ }^{\dagger}$, Zhuang Li, Xiaolei Han, Xingxing Liu, Guangyao Ran, Gangqi Wang, Daxin Pang, Xiaochun Tang*

Jilin Provincial Key Laboratory of Animal Embryo Engineering, College

of Animal Sciences, Jilin University, Jilin 130062, China

Tel: +86-431-8783-5175; Fax: +86-431-8798-0131

E-mail: xiaochuntang@jlu.edu.cn

Yaping Tian

Department of Dermatology and Venerology, 1st Hospital, Jilin University, Jilin 130062, China

Huan Wang

Department of Bioengineering, Jilin Agriculture Science and Technology

College, Jilin 132101, China

${ }^{\dagger}$ These authors contributed equally to this work. 